\title{
Upregulation of interleukin-17F in colorectal cancer promotes tumor invasion by inducing epithelial-mesenchymal transition
}

\author{
YUSHENG CHEN ${ }^{*}$, ZHOU YANG ${ }^{*}$, DEJUN WU, ZHIJUN MIN and YINGJUN QUAN \\ Department of General Surgery, Shanghai Pudong Hospital, Fudan University Pudong Medical Center, \\ Pudong, Shanghai 201399, P.R. China
}

Received January 15, 2019; Accepted June 7, 2019

DOI: 10.3892/or.2019.7220

\begin{abstract}
Interleukin-17F (IL-17F) is a member of the IL-17 family of proteins. Previous studies concerning IL-17F have mainly focused on its proinflammatory responses, whereas the present study explored its role as an oncogene in colorectal cancer (CRC). The present study investigated the expression of IL-17F in 69 patients with CRC. IL-17F was significantly overexpressed in tumor mucosa compared with that in the paired non-tumor mucosa. Furthermore, several studies from Gene Expression Omnibus (GEO) databases were analyzed to assess the association between IL-17F and overall survival and relapse-free survival time. Recombinant human IL-17F protein (rhIL-17F) and anti-IL-17F antibody were used to study the effect of IL-17F on the CRC cell line HCT-116 in vitro. According to the results of Transwell and wound healing assays, rhIL-17F promoted HCT-116 cell migration and invasion which was mediated by inducing epithelial-mesenchymal transition (EMT), whereas anti-IL-17F inhibited EMT.
\end{abstract}

\section{Introduction}

Previous reports have revealed that chronic inflammation can promote the occurrence, growth and metastasis of cancer. It is now widely accepted that acute inflammation will develop into chronic inflammation if not controlled in time, and chronic inflammation may increase the risk of cancer. Epidemiological studies have confirmed that $25 \%$ of cancers develop from inflammation. For example, patients with

Correspondence to: Dr Yingjun Quan or Dr Zhijun Min, Department of General Surgery, Shanghai Pudong Hospital, Fudan University Pudong Medical Center, 2800 Gongwei Road, Huinan, Pudong, Shanghai 201399, P.R. China

E-mail: qyjasmine@126.com

E-mail: minzhijun@126.com

${ }^{*}$ Contributed equally

Key words: interleukin-17F, colorectal cancer, epithelial-mesenchymal transition ulcerative colitis are 10 times more likely to develop colon cancer than normal individuals, and data show that $20 \%$ of patients with ulcerative colitis develop colon cancer 30 years later (1). The accumulation of inflammatory cells and inflammatory factors in the tumor microenvironment can promote angiogenesis, proliferation and metastasis of malignant cells, promote epithelial-mesenchymal transition (EMT) and reverse acquired immune response (2). It also alters the sensitivity of tumor cells to hormones and chemotherapeutic drugs.

Furthermore, cytokines serve an important role in the development of inflammation. Cytokines can be divided into lymphokines produced by lymphocytes and monocytes produced by monocytes and macrophages. Interleukin (IL), interferon, colony stimulating factor, tumor necrosis factor and transforming growth factor are known cytokines produced by immune cells. Cytokines in the tumor microenvironment usually serve an antitumor role, but in chronic inflammation, abnormal expression of cytokines can promote the transformation of surrounding normal cells into cancer cells in the early stage of cancer, and promote the growth and development of cancer cells (3).

Among them, $I L-17 F$ is located on chromosome $6 \mathrm{p} 12$ with a total length of 7,742 bp. It consists of 3 exons and 2 introns. IL-17F is produced by $\mathrm{T}$ helper (Th) 17 cells (4). Th17 cells associate innate immunity with adaptive immunity via chemokines and induce other Th cell subsets to aggregate at the site of infection in the late stage of inflammation. As a prototype cytokine of the IL-17 family, IL-17F induces different tissues and cells to produce inflammatory cytokines, chemokines and metalloproteinases, and collects centralized granulocytes from tissues. Current research results have confirmed that Th17 cytokines such as IL-17F can promote the synthesis of inflammatory cells, increase the levels of chemokines and promote the expression of tissue degradation matrix metalloproteinases, thus causing tissue damage (5). However, there are few studies on the association between IL-17F and cancer, particularly colorectal cancer (CRC). In the present study, we found that $I L-17 F$ gene is abnormally expressed in CRC tissues. However, the mechanism by which IL-17F is activated and has an effect on CRC tissues remains unclear. Therefore, it is of great significance to study the differential expression of the IL-17F gene in CRC, as well as the molecular mechanism and signaling pathway in the development of CRC. 


\section{Materials and methods}

Patients and specimens. CRC tumor specimens and paired non-tumor mucosa were collected from July 2012 to July 2018 in Shanghai Pudong Hospital. Patients with the following criteria were excluded from participation: Patients that had received adjuvant chemotherapy or radiotherapy prior to surgery or had been diagnosed with additional cancer types. All patients were classified according to the 7th edition of the Tumor-Node-Metastasis (TNM) staging system (www.cancerstaging.org). Postoperative adjuvant therapies were performed according to standard schedules and doses. All participating patients provided their written informed consent. The present study was approved by the Ethics Committee of Shanghai Pudong Hospital. Patients' data was shown in Table I.

Immunohistochemical (IHC) staining. IHC staining was carried out according to the manufacturer's instructions (IHC assay kit; ProteinTech Group, Inc.). Briefly, formalin-fixed and paraffin-embedded tissue sections were deparaffinized in xylene and hydrated with decreasing concentrations of ethanol (100, 95, 80 and $75 \%)$. The slices were then soaked in $10 \%$ BSA to inhibit endogenous peroxidase activity and incubated with the anti-IL-17F rabbit polyclonal antibody (dilution 1:100; $\mathrm{AB} \_354739, \mathrm{R} \& \mathrm{D}$ Systems, Inc.) at $4^{\circ} \mathrm{C}$ overnight. A horseradish peroxidase (HRP)-conjugated rabbit secondary antibody (dilution 1:4,000; ProteinTech Group, Inc.) was added for $60 \mathrm{~min}$ at room temperature; then, 3,3'-diaminobenzidine development (DAB Substrate Chromogen System; Dako; Agilent Technologies, Inc.) and hematoxylin staining were performed according to standard protocols. Slides were fixed and images were obtained with an Olympus IX71 inverted microscope and a DP2-BSW Olympus image acquisition software system. IHC staining was scored based on the proportion of cell staining and the staining intensity. The percentage of positive cells was divided into 5 grades (percentage scores): $<10 \%$ (0), $10-25 \%$ (1), $>25-50 \%$ (2), $>50-75 \%$ (3) and $>75 \%$ (4). The intensity of staining was divided into 4 grades (intensity scores): No staining, 0 ; weak staining, 1; moderate staining, 2; strong staining, 3. IL-17F staining positivity was determined by the following formula: Overall score=percentage score $\mathrm{x}$ intensity score. An overall score $\leq 3$ was defined as negative and $>3$ was defined as positive. IL-17F staining was scored by two independent researchers who were blinded to the clinical characteristics of the patients.

Drug treatment and experimental design. Recombinant human IL-17F protein (rhIL-17F) and the anti-IL-17F antibody were purchased from R\&D Systems, Inc. (1335-IL-025, MAB13352). The working concentration of rhIL-17F and anti-IL-17F antibody were 100 and $500 \mathrm{nM}$, respectively. The experiment was divided into 3 groups: Negative control (NC) group, rhIL-17F group and anti-IL-17F group.

Cell line and cell culture. The human CRC cell line HCT-116 (CVCL_0291) was purchased from the University of Colorado Cancer Center Cell Bank and was cultured in RPMI-1640 medium supplemented with 10\% FBS (Invitrogen; Thermo Fisher Scientific, Inc.) at $37^{\circ} \mathrm{C}$ in a $5 \% \mathrm{CO}_{2}$ atmosphere. Cells were digested and passaged when cell fusion reached $80 \%$.
Protein extraction and western blot analysis. Total protein was extracted using RIPA lysis buffer with $1 \%$ phenylmethanesulfonyl fluoride. Then, equal amounts $(20 \mu \mathrm{g})$ of protein, as determined with a bicinchoninic acid protein assay kit (Thermo Fisher Scientific, Inc.) were separated by $10 \%$ SDS-PAGE. The proteins were then transferred to PVDF membranes (0.45-mm; Beijing Solarbio Science \& Technology Co., Ltd.). The membranes were blocked with 5\% non-fat milk for $1 \mathrm{~h}$ at room temperature and then incubated with primary antibodies at $4^{\circ} \mathrm{C}$ for $12 \mathrm{~h}$. The following antibodies were employed: Anti-E-cadherin (AB_10697811), anti-Twist1 (AB_10598006), anti-vimentin (AB_2273020), anti-Snail1 (13099-1) (dilution 1:1,000; ProteinTech Group, Inc.) and anti-IL-17F (dilution 1:1,000; R\&D Systems, Inc.) rabbit polyclonal antibodies. Anti-GAPDH rabbit polyclonal antibodies (dilution 1:4,000; AB_2107436, ProteinTech Group, Inc.) were used as loading controls for normalization. The secondary antibodies were anti-rabbit antibodies conjugated to HRP (dilution 1:4,000; AB_2722564, ProteinTech Group, Inc.). The antibodies were used at 1:4,000 dilution and were incubated for $\sim 1 \mathrm{~h}$ at room temperature. The bands were visualized with ECL reagents (Thermo Fisher Scientific, Inc.) and developed by Omega Lum $^{\mathrm{TM}} \mathrm{G}$ (Aplegen Life Science).

Immunofluorescence $(I F)$. Coverslips were placed horizontally on the bottom of a 6-well plate upon cleaning, disinfection and 24-h ultraviolet irradiation. Then, the cells were seeded on the coverslips at a density of $1 \times 10^{6}$ cells per well and cultured in an incubator. The coverslips were rinsed with PBS for 5 min 3 times and fixed with $4 \%$ paraformaldehyde for $15 \mathrm{~min}$, followed by permeabilization of the cells with $0.3 \%$ Triton X-100 for additional $10 \mathrm{~min}$. Next, the coverslips were rinsed with PBS again for 5 min 3 times and blocked by incubating the cells in 5\% BSA for $60 \mathrm{~min}$. Then, the cells were stained with anti-E-cadherin rabbit polyclonal antibody (dilution 1:100; ProteinTech Group, Inc.), followed by a 12-h incubation period at $4^{\circ} \mathrm{C}$. After washing the unbound antibody with PBS for 5 min 3 times, the cells were incubated with an Alexa 488-conjugated goat anti-rabbit immunoglobulin G secondary antibody (1:1,000; Cell Signaling Technology, Inc.) for $1 \mathrm{~h}$ at $4^{\circ} \mathrm{C}$ in the darkness. Finally, DAPI was used as a counterstain to label the nuclei. The stained cells were then acquired and photographed with a fluorescence microscope.

$R N A$ extraction and reverse transcription-quantitative $P C R$ $(R T-q P C R)$. Total RNA was extracted with TRIzol reagent (Invitrogen; Thermo Fisher Scientific, Inc.). Complementary DNA was obtained from total RNA using PrimeScript ${ }^{\mathrm{TM}}$ RT Reagent kit (Takara Bio, Inc.). The expression of mRNA was assessed by RT-qPCR, which was carried out in triplicate using a SYBR Premix Ex Taq ${ }^{\mathrm{TM}}$ kit (Takara Bio, Inc.) and an ABI 7900HT Real-Time PCR System (Applied Biosystems; Thermo Fisher Scientific, Inc.). The primers used are shown in Table II. The comparative quantification cycle threshold values $\left(2^{-\Delta \Delta C q}\right)$ were employed to analyze the results (6).

Cell proliferation assay. For this assay, $5 \times 10^{3}$ cells were seeded into 96-well plates and incubated for the following times: 0, 24, 48 and 72 h. Next, $10 \mu$ l Cell Counting Kit-8 (CCK-8; Dojindo Molecular Technologies, Inc.) solution was added to 
Table I. Clinical characteristics of the colorectal cancer patients $(\mathrm{N}=67)$.

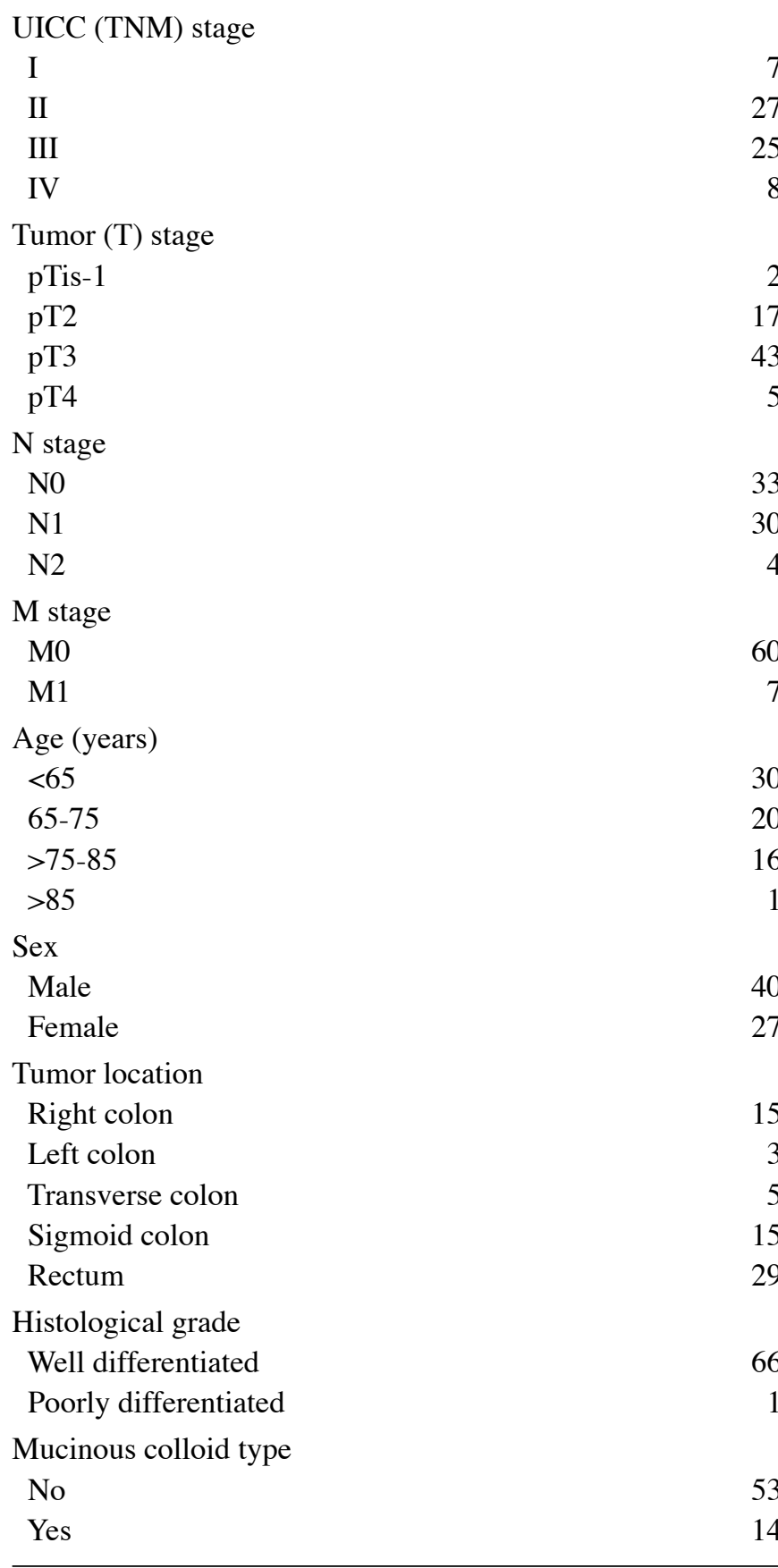

each well of the plate, and incubation was continued for $2 \mathrm{~h}$. Finally, the absorbance of each well at a $450-\mathrm{nm}$ wavelength was measured.

Colony formation assay. For this assay, 500 cells were seeded into 6-well plates and incubated at $37^{\circ} \mathrm{C}$. Colony size was observed daily under a microscope until the number of cells in the majority of colonies was $>50$. Then, the medium was removed and the cells were stained with $0.2 \%$ crystal violet for $30 \mathrm{~min}$. The cells were washed 3 times with PBS, and then photographed and the colonies were counted. The percentage of colony formation was calculated using the following equation: Percentage of colony formation $(\%)=$ colony number/500 x 100 .
Cell migration and invasion assays. Cell migration and invasion capacities were analyzed with Transwell plates (24-well insert, $8-\mu \mathrm{m}$ pore size; BD Biosciences). The filters (Corning Inc.) were coated with (invasion assay) or without (migration assay) $55 \mu 1$ Matrigel (1:8 dilution; BD Biosciences). For the migration assays, $5 \times 10^{4}$ cells were suspended in $200 \mu \mathrm{l}$ serum-free medium and seeded into the Matrigel-uncoated upper chambers. Then, $600 \mu \mathrm{l}$ medium containing 10\% FBS was added to the lower chamber as a chemoattractant. After incubation at $37^{\circ} \mathrm{C}$ for $24 \mathrm{~h}$, the membranes were fixed with $4 \%$ formaldehyde for $30 \mathrm{~min}$ and stained with $0.1 \%$ crystal violet at room temperature for $30 \mathrm{~min}$. For invasion assays, $1 \times 10^{5}$ cells suspended in $200 \mu 1$ serum-free medium were seeded into the Matrigel-coated upper chambers. The rest of the protocol was identical to that described above. The cells were counted and photographed under an inverted microscope (x400) over 5 different fields for each triplicate filter.

Wound healing assay. For this assay, $5 \times 10^{5}$ cells were seeded into 6 -well plates and cultured at $37^{\circ} \mathrm{C}$ for $24 \mathrm{~h}$. A $200-\mu 1$ sterile micropipette tip was used to scratch the confluent monolayers in a straight line when cells were $80-90 \%$ confluent. Then, floating cells were washed with PBS 3 times, and the cell culture was continued to upon changing the complete medium to serum-free medium. Images of the same wound position were captured at 0 and $48 \mathrm{~h}$ under an inverted microscope (x200). The migration results were evaluated by Image J software (National Institutes of Health, Bethesda, MD, USA).

Statistical analysis. SPSS software (version 19.0; IBM Corp.) was used for statistical analysis of all the experimental data. GraphPad Prism (version 7; GraphPad Software, Inc.) was used to determine the statistical results. All data are expressed as the mean \pm standard deviation. Statistical analysis of the data derived from 2 groups was performed with the Student's t-test. Comparisons of multiple groups were performed by one-way analysis of variance (ANOVA) followed by Least Significant Difference test. For survival analysis, PROGgeneV2 (http://watson.compbio.iupui.edu/chirayu/proggene) website tools was used to analyze the overall survival (OS) and relapse-free survival (RFS) of the patients with CRC based on Gene Expression Omnibus (GEO) databases (accession nos. GSE14333, GSE17536, GSE16125 and GSE29621) (7-10). $\mathrm{P}<0.05$ was considered to indicate a statistically significant difference.

\section{Results}

IL-17F is upregulated in CRC tumor tissues. In order to analyze the function of IL-17F in CRC, RT-qPCR was performed to detect IL-17F (Fig. 1A). Significant overexpression of IL-17F in CRC tumor tissues compared with that noted in the paired non-tumor mucosa was observed. Western blotting (Fig. 1B) and IHC (Fig. 1C) were used to detect the protein expression of IL-17F, and the same trend was observed. Thus, upregulation of IL-17F was observed in tumor tissues at both the mRNA and protein levels.

$I L-17 F$ influences the overall survival $(O S)$ and relapse-free survival (RFS) of patients with CRC. To explore the clinical 
Table II. Primers for RT-qPCR.

\begin{tabular}{lll}
\hline Gene & \multicolumn{1}{c}{ Forward primer } & \multicolumn{1}{c}{ Reverse primer } \\
\hline IL-17F & GCTGTCGATATTGGGGCTTG & GGAAACGCGCTGGTTTTCAT \\
Actin & GGGACCTGACTGACTACCTC & TCATACTCCTGCTTGCTGAT \\
E-cadherin & AGTCACTGACACCAACGATAAT & ATCGTTGTTCACTGGATTTGTG \\
Vimentin & AGTCCACTGAGTACCGGAGAC & CATTTCACGCATCTGGCGTTC \\
Snail1 & AAGGATCTCCAGGCTCGAAAG & GCTTCGGATGTGCATCTTGA \\
Twist 1 & GTACATCGACTTCCTCTACCAG & CATCCTCCAGACCGAGAAG \\
\hline
\end{tabular}

A

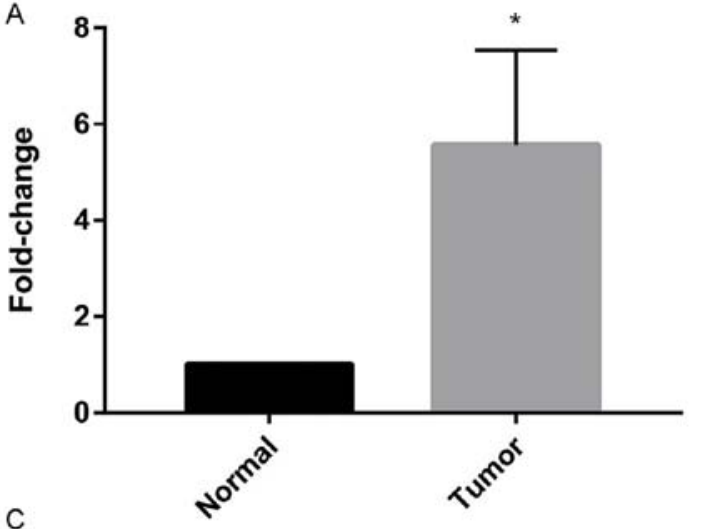

IL-17F

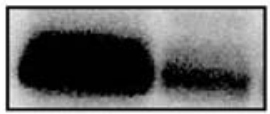

B-actin

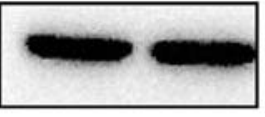

Normal Tumor
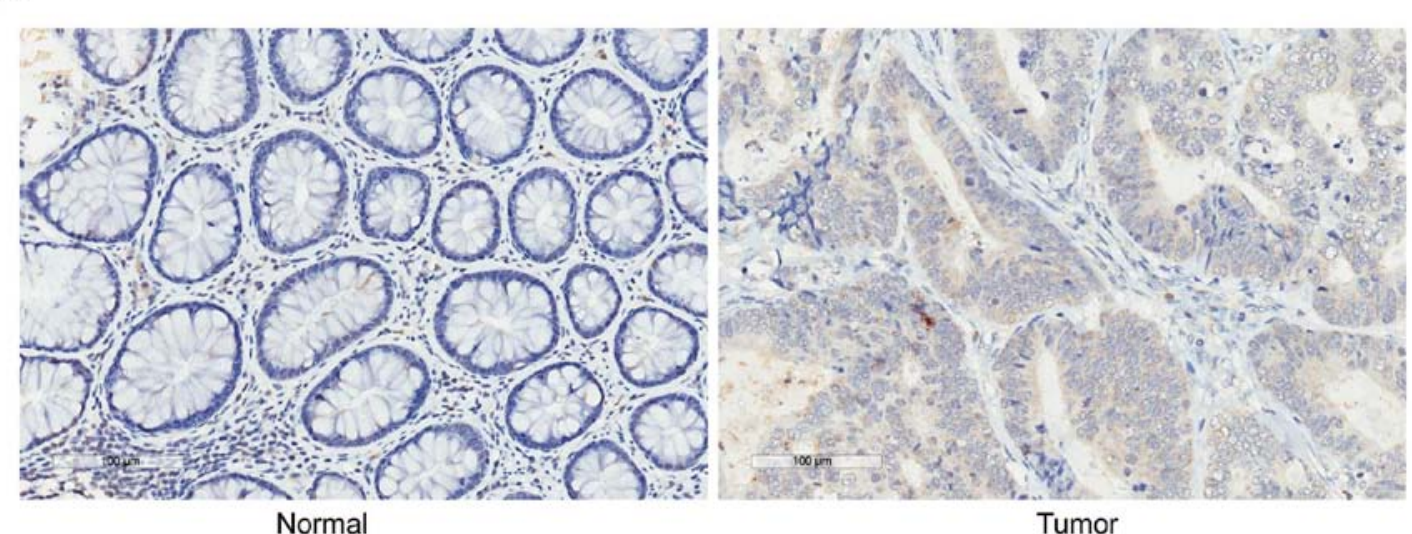

Figure 1. IL-17F is overexpressed in colorectal cancer. (A) Expression of common IL-17F was detected by reverse transcription-quantitative PCR. "P $<0.05$, compared to the normal tissues. (B) Expression of IL-17F protein was detected by western blotting. (C) Expression of IL-17F protein was detected by immunohistochemistry. IL-17F, interleukin-17F.

significance of the upregulation of IL-17F in CRC, the online tool PROGgeneV2 (http://watson.compbio.iupui. edu/chirayu/proggene) was used to analyze the OS and RFS of patients with CRC based on Gene Expression Omnibus (GEO) databases (6) (Fig. 2). In GSE14333, overexpression of IL-17F was associated with worse RFS [hazard ratio (HR), 0.17, P=0.03]; in GSE17536, GSE16125 and GSE29621, overexpression of IL-17F was associated with worse OS (HR, 0.3, $\mathrm{P}=0.045 ; \mathrm{HR}, 0.3, \mathrm{P}=0.02$; and $\mathrm{HR}, 0.1, \mathrm{P}=0.014$, respectively). The data indicated that IL-17F was a prognostic predictor of OS and RFS in patients with CRC.

IL-17F promotes CRC invasion and migration in vitro. Transwell assays were used to evaluate the migration and invasion capabilities of HCT-116 cells (Fig. 3A). There were more migrated and invasive cells in the rhIL-17F group than these numbers in the NC group in the migration and invasion assays, while the group treated with anti-IL-17F antibody exhibited fewer cells compared with the NC group. In the wound healing assay (Fig. 3B), the rhIL-17F group exhibited a smaller wound area after $48 \mathrm{~h}$, whereas the anti-IL-17F group exhibited a larger area. Collectively, these results indicated that IL-17F promotes HCT-116 cell invasion and migration.

Anti-IL-17F antibody inhibits the colony formation of CRC cells. The colony formation assay was performed to assess the influence of IL-17F on HCT-116 colony formation ability (Fig. 4A and B). There was no difference in the percentage of colony formation between the NC group and the rhIL-17F group, whereas the anti-IL-17F group exhibited a significantly 

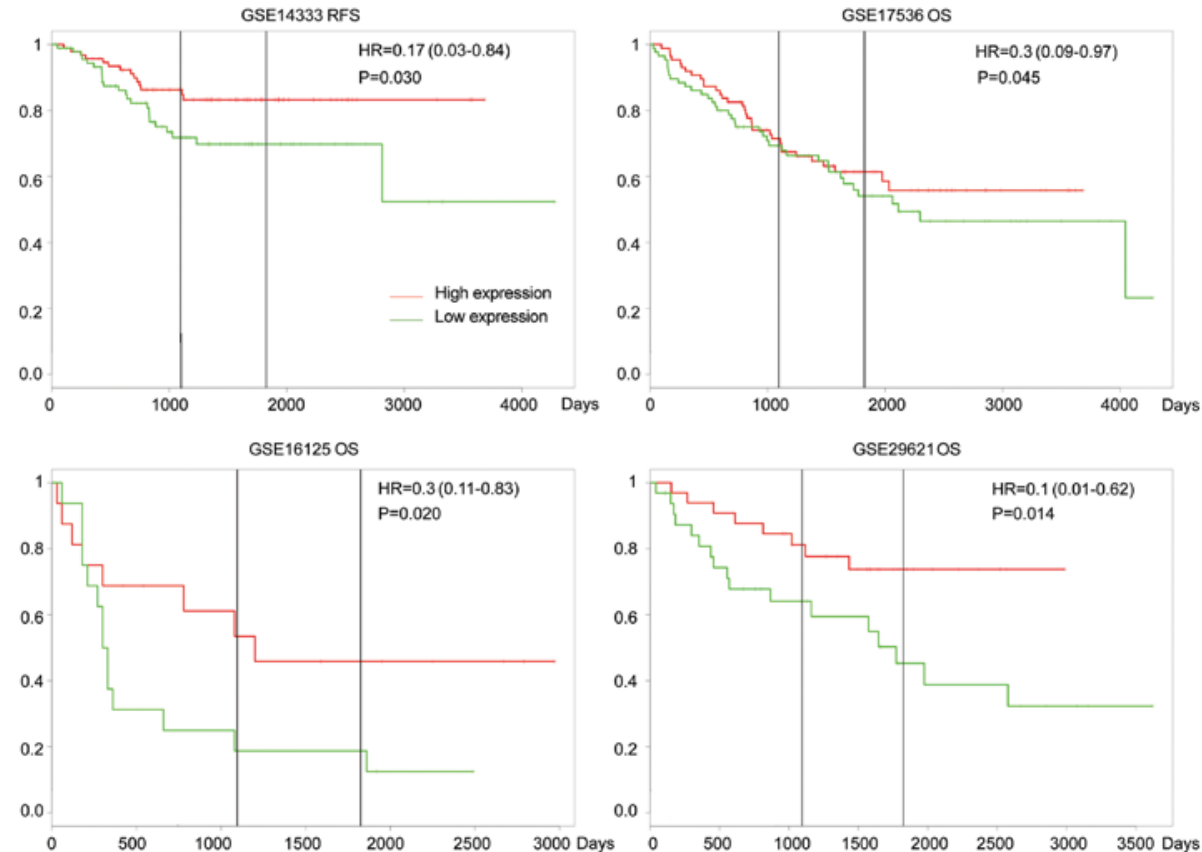

Figure 2. Overall survival (OS) and relapse-free survival (RFS) of patients with colorectal cancer based on high and low expression of IL-17F in different Gene Expression Omnibus databases (GSE14333, GSE17536, GSE16125 and GSE29621), as analyzed with the PROGgeneV2 online tool. HR, hazard ratio. IL-17F, interleukin-17F.

A

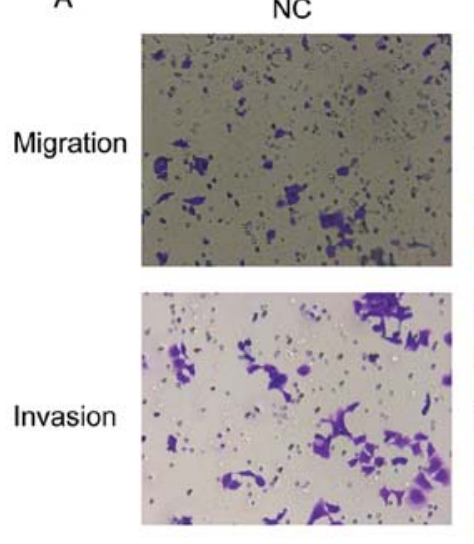

B
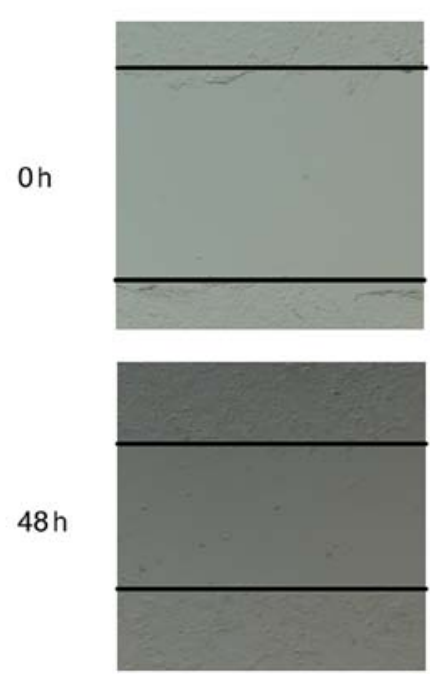

rhIL-17F
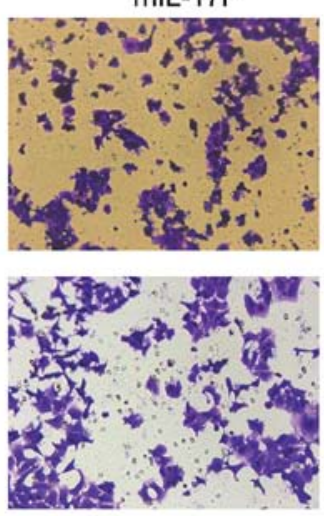

rhIL-17F
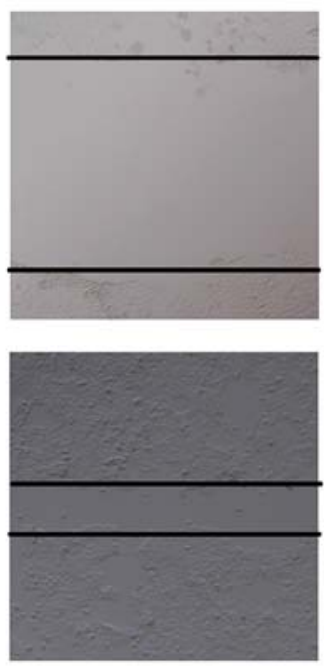

antilL-17F
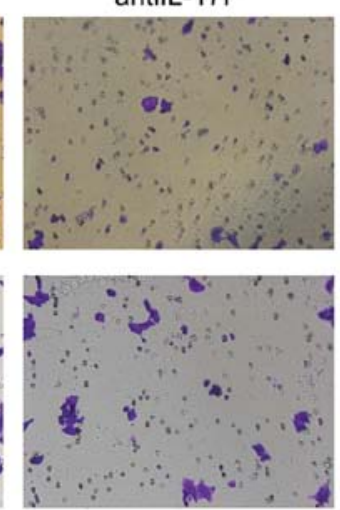

antill-17F
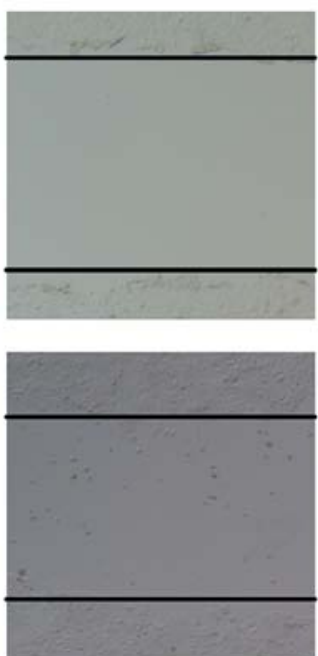
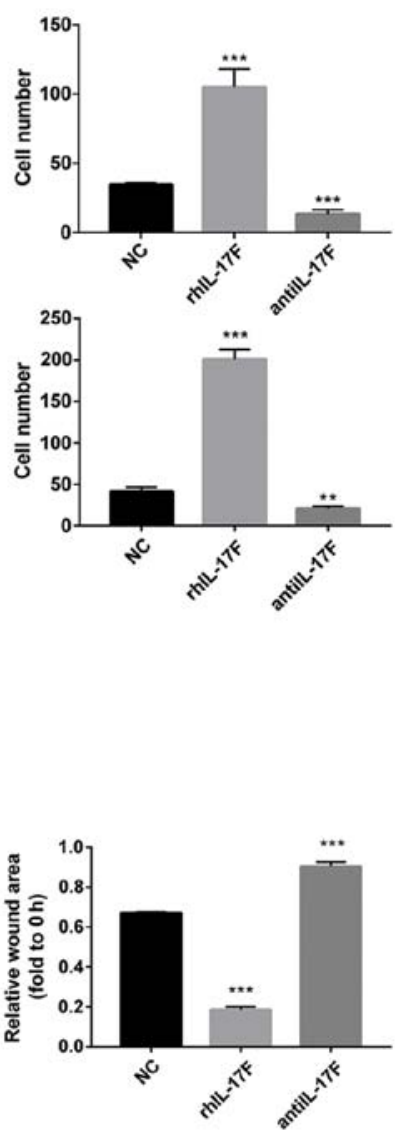

Figure 3. (A) rhIL-17F promotes HCT-116 cell invasion and migration according to the results of Transwell migration and invasion assays. (B) rhIL-17F promotes HCT-116 cell migration according to the results of the wound healing assay. ${ }^{* *} \mathrm{P}<0.01,{ }^{* * * *} \mathrm{P}<0.001$, compared with the negative control (NC) group. rhIL-17F, recombinant human interleukin-17F. 
A NC
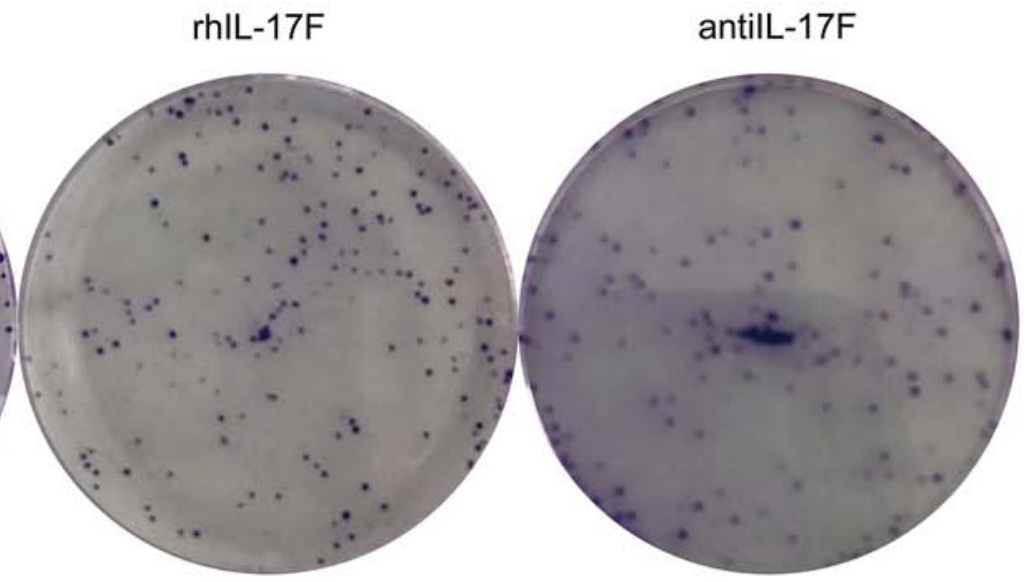

B

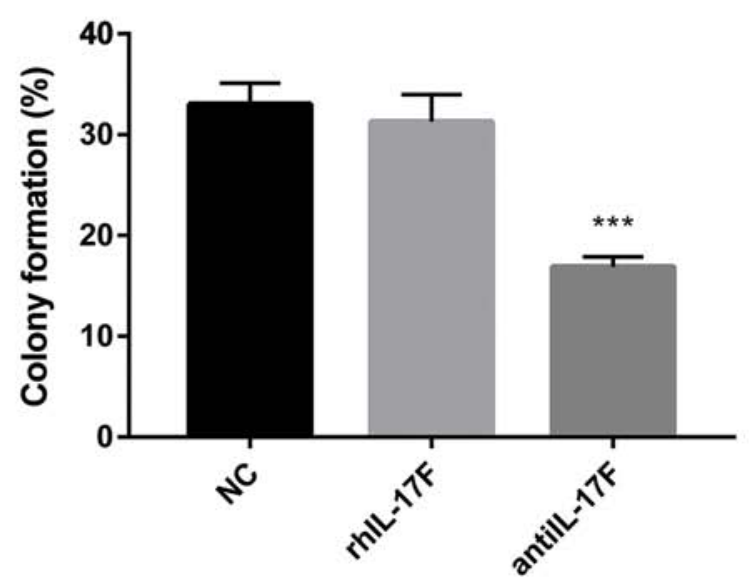

C

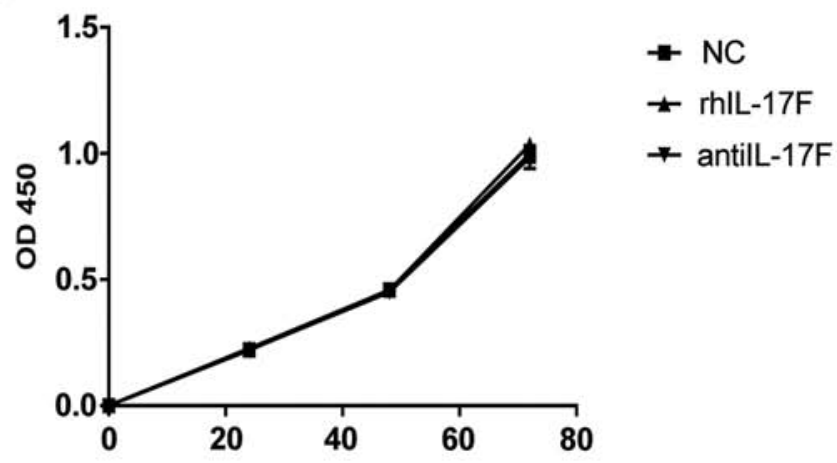

Figure 4. (A) Colony formation assay for each group. (B) The group treated with anti-interleukin-17F antibody exhibited decreased percentage of colony formation compared with the negative control (NC) group. ${ }^{* * *} \mathrm{P}<0.001$. (C) Cell proliferation was detected by Cell Counting Kit- 8 assay, and no difference was observed.,. rhIL-17F, recombinant human interleukin-17F.

inhibited colony formation ability compared with the NC group. In addition, CCK-8 assay was further performed to detect cell proliferation (Fig. 4C), but no difference was identified between each group. These results indicated that rhIL-17F had no effect on the colony formation ability or proliferation of HCT-116 cells.

IL- $17 F$ induces EMT in CRC cells. RT-qPCR was performed to detect the mRNA expression of several main EMT-associated markers (Fig. 5A). E-cadherin was significantly decreased in the rhIL-17F group compared with the NC group, whereas vimentin, Snail1 and Twist1 were significantly upregulated. Notably, the anti-IL-17F group exhibited the opposite changes. Furthermore, western blotting was performed to detect the protein expression of EMT-associated markers (Fig. 5B). The changing trend was in agreement with the results of RT-qPCR. Next, the location and expression of E-cadherin were assessed by IF (Fig. 5C). E-cadherin was located in the cell membrane and cytoplasm, and the changing trend in expression was in agreement with the results of western blotting and RT-qPCR.

\section{Discussion}

IL-17F is member of the IL-17 family. Previous research concerning IL-17 focused on inflammation and immunity (11).
Inflammation is closely associated with cancer, as recent studies have demonstrated the influence of IL-17 on different tumors. In non-small cell lung cancer (NSCLC), IL-17 was found to increase the net angiogenic activity and in vivo growth of NSCLC by promoting CXCR-2-dependent angiogenesis (12). In early-stage breast cancer (13), IL-17BR was found to serve as a prognostic factor. However, the number of studies which have focused on the association between IL-17F and cancer, particularly colorectal cancer (CRC), is limited.

The present study observed a significant upregulation of IL-17F in tumor tissue compared with that noted in paired non-tumor mucosa. Furthermore, overexpression of IL-17F was associated with worse overall survival (OS) and relapse-free survival (RFS) according to GEO databases. IL is a secretory protein. Therefore, rhIL-17F was used to study its effect in vitro. rhIL-17F obviously promoted HCT-116 cell invasion and migration in vitro according to the results of Transwell and wound healing assays.

It is known that epithelial-mesenchymal transition (EMT) is a key mechanism associated with cancer invasion. EMT refers to the transformation of epithelial cells into mesenchymal cells under specific physiological and pathological conditions (14). The concept of EMT was first proposed in the field of embryonic development. Since then, numerous studies on EMT have been carried out, involving different 
A

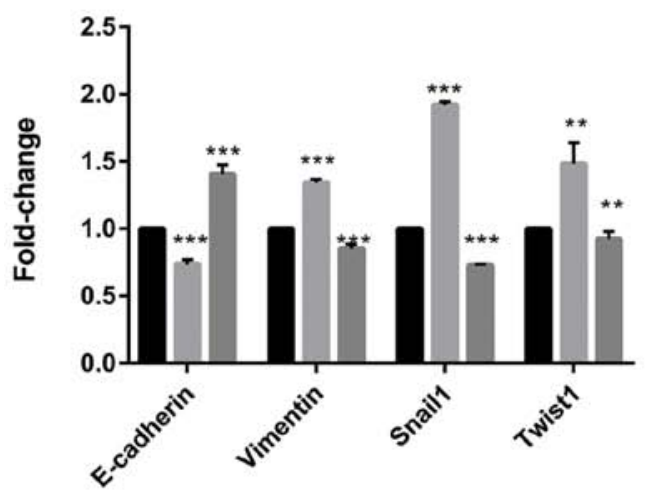

C

$\mathrm{NC}$
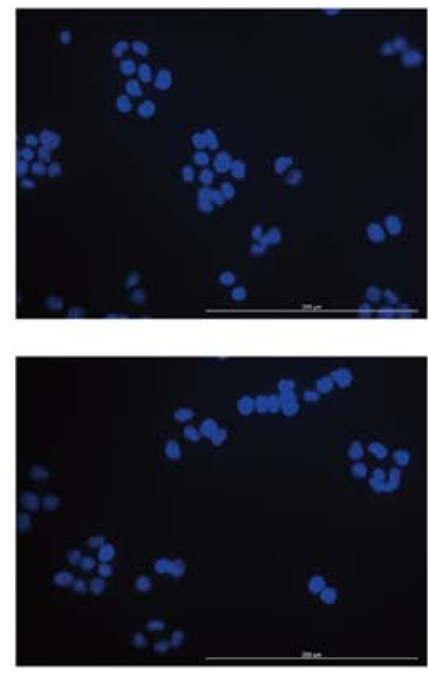

rhIL-17F

antilL-17F

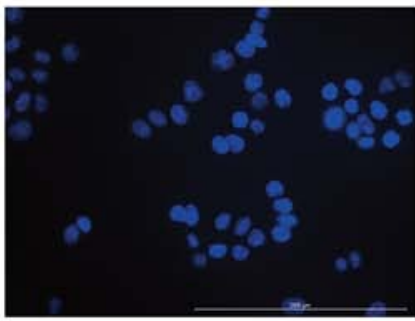

B

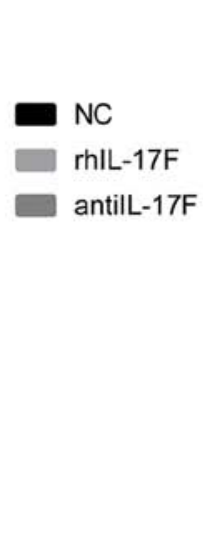

E-cadherin

Vimentin

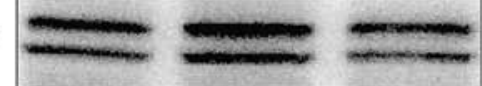

Snail1

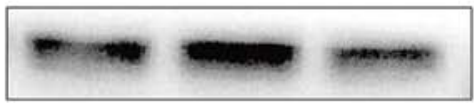

Twist1

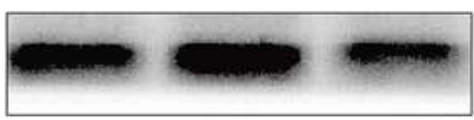

$\beta$-actin

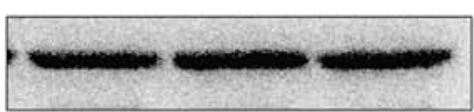

E-cadherin
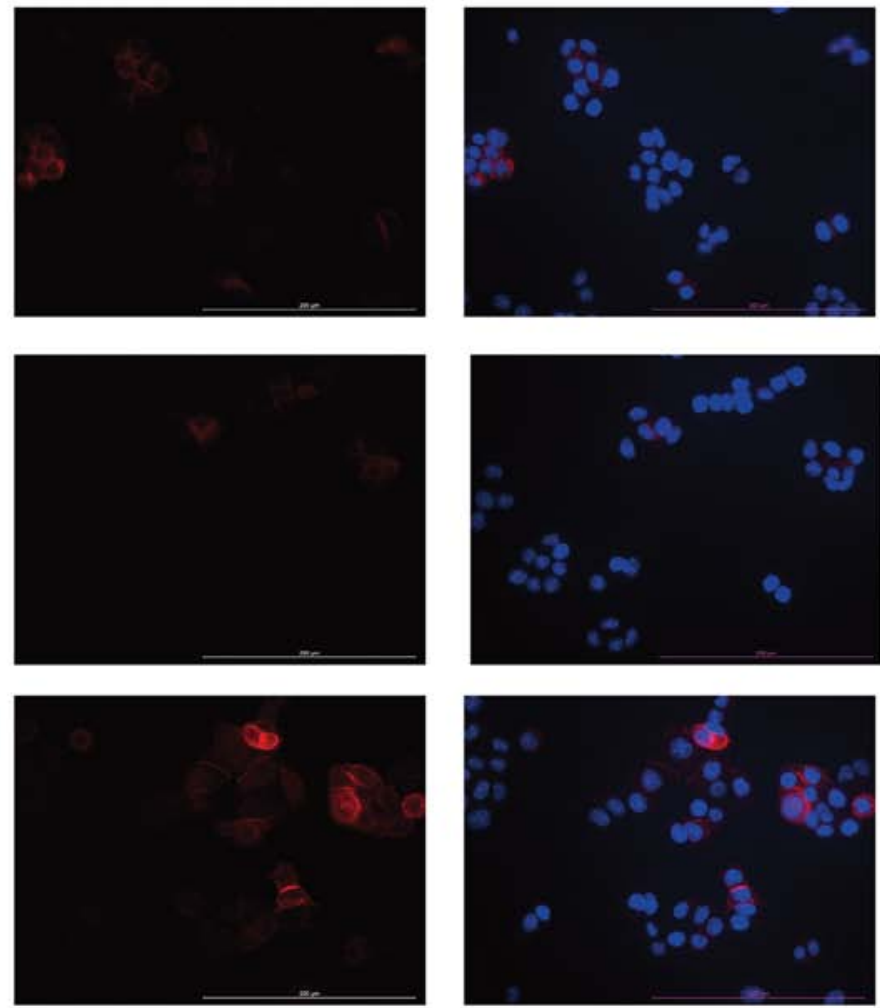

Figure 5. EMT-associated markers were detected by (A) reverse transcription-quantitative PCR and (B) western blotting. ${ }^{* *} \mathrm{P}<0.01,{ }^{* * *} \mathrm{P}<0.001$, compared with the negative control (NC) group. (C) E-cadherin location and expression were detected by immunofluorescence. EMT, epithelial-mesenchymal transition; rhIL-17F, recombinant human interleukin-17F.

life phenomena and pathological processes (15). During EMT, intercellular junctions and cell polarity disappear, epithelial markers are downregulated, mesenchymal phenotypes and associated markers are gradually upregulated, and the biological behavior of cells also changes, which is characterized by enhanced migration and invasion abilities (16). This process is accompanied by decreased E-cadherin expression and concurrent increases in the expression of vimentin, $\mathrm{N}$-cadherin and the transcription regulators Twist and Snail. These key EMT-associated markers were further detected in our study. rhIL-17F significantly decreased the expression of E-cadherin and increased the expression of vimentin, Snail1, Twist1; whereas the anti-IL-17F antibody produced the opposite effect. This result indicates that IL-17F promotes HCT-116 cell invasion and migration by inducing EMT. There are some shortcomings in our study, as we only researched the effect of IL-17F on EMT in vivo. In fact, IL-17F polymorphism is also closely related to the occurrence and development of various tumors, which will be our next research focus.

Taken together, the present study demonstrated that IL-17F was upregulated in CRC tumor tissues and was associated worse OS and RFS of CRC patients. In vitro, IL-17F promoted CRC cell invasion and migration by inducing EMT. 


\section{Acknowledgements}

Not applicable.

\section{Funding}

The present study was funded by Puxiu Medical Talents Training Program of Pudong Hospital (grant no. PX201702).

\section{Availability of data and materials}

The datasets used and analyzed during the present study are available from the corresponding author on reasonable request.

\section{Authors' contributions}

YC and ZY performed the experiments and wrote the manuscript. DW contributed to the statistical analysis of the data and collected tissues. YQ and ZM conceived and designed the study. All authors read and approved the manuscript and agree to be accountable for all aspects of the research in ensuring that the accuracy or integrity of any part of the work are appropriately investigated and resolved.

\section{Ethics approval and consent to participate}

All procedures involving human participants were performed in accordance with the Ethics Committee of Shanghai Pudong Hospital and with the 1964 Declaration of Helsinki and its later amendments or comparable ethical standards. All patients provided their written informed consent. The study protocol was approved by the Pudong Hospital Committee on human research.

\section{Patient consent for publication}

Not applicable.

\section{Competing interests}

The authors declare that they have no competing interests.

\section{References}

1. Long AG, Lundsmith ET and Hamilton KE: Inflammation and colorectal cancer. Curr Colorectal Cancer Rep 13: 341-351, 2017.
2. Song J, Feng L, Zhong R, Xia Z, Zhang L, Cui L, Yan H, Jia X and Zhang Z: Icariside II inhibits the EMT of NSCLC cells in inflammatory microenvironment via down-regulation of Akt/NF- $\kappa \mathrm{B}$ signaling pathway. Mol Carcinog 56: 36-48, 2017.

3. Taniguchi K and Karin M: IL-6 and related cytokines as the critical lynchpins between inflammation and cancer. Semin Immunol 26: 54-74, 2014.

4. Conti HR, Shen F, Nayyar N, Stocum E, Sun JN, Lindemann MJ, Ho AW, Hai JH, Yu JJ, Jung WJ, et al: Th17/IL-17 receptor signaling and not Th1 cells are essential for mucosal host defense against oral candidiasis. Cytokine 206: 299-311, 2009.

5. Hymowitz SG, Filvaroff EH, Yin JP, Lee J, Cai L, Risser P, Maruoka M, Mao W, Foster J, Kelley RF, et al: IL-17s adopt a cystine knot fold: Structure and activity of a novel cytokine, IL-17F, and implications for receptor binding. EMBO J 20: 5332-5341, 2001.

6. Schmittgen TD: Real-time quantitative PCR. Methods 25: 383-385, 2001.

7. Jorissen RN, Gibbs P, Christie M, Prakash S, Lipton L, Desai J, Kerr D, Aaltonen LA, Arango D, Kruhoffer M, et al: Metastasis-associated gene expression changes predict poor outcomes in patients with dukes stage B and $\mathrm{C}$ colorectal cancer. Clin Cancer Res 15: 7642-7651, 2009.

8. Smith JJ, Deane NG, Fei WU, Merchant NB, Zhang B, Jiang A, Pengcheng LU, Johnson JC, Schmidt C, Bailey CE, et al: Experimentally derived metastasis gene expression profile predicts recurrence and death in patients with colon cancer. Gastroenterology 138: 958-968, 2010.

9. Reid JF, Gariboldi M, Sokolova V, Capobianco P, Lampis A, Perrone F, Signoroni S, Costa A, Leo E, Pilotti S and Pierotti MA: Integrative approach for prioritizing cancer genes in sporadic colon cancer. Genes Chromosomes Cancer 48: 953-962, 2009.

10. Chen DT, Hernandez JM, Shibata D, McCarthy SM, Humphries LA, Clark W, Elahi A, Gruidl M, Coppola D and Yeatman T: Complementary strand microRNAs mediate acquisition of metastatic potential in colonic adenocarcinoma. J Gastrointest Surg 16: 905-913, 2012.

11. van der Waart AB, van der Velden WJ, Blijlevens NM and Dolstra H: Targeting the IL17 pathway for the prevention of graft-versus-host disease. Biol Blood Marrow Transplant 20: 752-759, 2014.

12. Numasaki M, Watanabe M, Suzuki T, Takahashi H, Nakamura A McAllister F, Hishinuma T, Goto J, Lotze MT, Kolls JK and Sasaki H: IL-17 enhances the net angiogenic activity and in vivo growth of human non-small cell lung cancer in SCID mice through promoting CXCR-2-dependent angiogenesis. J Immunol 175: 6177-6189, 2005.

13. Ma XJ, Hilsenbeck SG, Wang W, Ding L, Sgroi DC, Bender RA, Osborne CK, Allred DC and Erlander MG: The HOXB13:IL17BR expression index is a prognostic factor in early-stage breast cancer. J Clin Oncol 24: 4611-4619, 2006.

14. Thiery JP, Acloque H, Huang RY and Nieto MA: Epithelialmesenchymal transitions in development and disease. Cell 139: 871-890, 2009.

15. Nieto MA: Epithelial-mesenchymal transitions in development and disease: Old views and new perspectives. Int J Dev Biol 53: 1541-1547, 2009.

16. Lim J and Thiery JP: Epithelial-mesenchymal transitions: Insights from development. Development 139: 3471-3486, 2012. 